\title{
Transfer of grain calibrations between a handheld and a process instrument
}

\author{
F. Benozzo* and P. Berzaghi \\ Department of Animal Medicine, Production and Health University of Padova, Italy. E-mail: francesco.benozzo@unipd.it
}

Transfer of calibrations between instruments is a key issue to use the value of a calibration over multiple units. Transfer is relatively easy between spectrometers of the same type but can be problematic between different instrument models. Two hundred and seventy samples of wheat from Northern Italy were scanned using a Corona Extreme and an Aurora handheld NIR. Samples $(n=46)$ from three different locations were removed from the original dataset and used for external validation. The PLS calibrations performances were satisfactory, with SECV for Moisture of $0.09 \%$ and $0.13 \%$ and for Protein of $0.28 \%$ and $0.45 \%$, respectively for Aurora handheld NIR and Corona Extreme. Performance of validation (SEP) within instrument was of $0.07 \%$ and $0.11 \%$ for Moisture and of $0.27 \%$ and $0.37 \%$ for Protein, for the handheld and the process instrument, respectively. When the same calibrations were used to predict samples across instruments, the SEP was of $0.08 \%$ and $0.19 \%$ for Moisture and of $0.34 \%$ and $0.47 \%$ for Protein, for Corona Extreme predicting Aurora handheld NIR and vice versa, respectively. Both instruments can accurately predict the parameters of interest on wheat and could use the same calibration avoiding time-consuming standardization procedures.

\section{Introduction}

Industry has created the need for a cost effective and nondestructive quality-control analysis system. Several studies have demonstrated the ability of diode array spectrometers, working in transmission or reflectance, to accurately measure moisture and protein content in grain. The prediction accuracy for on-line measurements on combine harvesters was found comparable with that achieved on similar but stationary units proving the technical feasibility of estimating grain quality on the go with NIR technology. Over the last twenty years strong interest has increased for portable spectrometers leading to the development and marketing of handheld devices that enable new applications especially in the agrifeed industry. Handheld NIR spectrometers are powerful instruments offering several advantages for nondestructive in situ analysis ${ }^{1}$ but, since they are relatively new, the investment in developing new calibrations may discourage or slow down their use. Transfer of calibrations between near infrared instruments is not always straightforward, even when the instruments are nominally the same. Problems can include both wavelength shifts and differences in absorbance response between instruments. ${ }^{2}$

\section{Materials and methods}

\section{Samples and reference data}

During the 2016 harvesting 270 samples of wheat were collected from different locations in the area of the Po Valley. Samples of red (185) and durum (85) wheat were analyzed with a scanning monochromator (Infratec 1241, Foss Italy S.r.l., Italy) for Moisture and Crude Protein (Table 1).

\section{Spectrometers}

Two diode array spectrometers were evaluated: Corona Extreme (Carl Zeiss Spectroscopy GmbH, Germany) and a Auroranir (GraiNit S.r.l., Italy). Both instruments operate in reflectance mode and use a diode array sensor (256 pixels) in the range of $960-1650 \mathrm{~nm}$. They differ in optical

\section{Correspondence}

F. Benozzo (francesco.benozzo@unipd.it)

doi: $10.1255 /$ nir2017.011

Citation: F. Benozzo and P. Berzaghi, "Transfer of grain calibrations be-

tween a handheld and a process instrument", in Proc. $18^{\text {th }}$ Int. Conf. Near

Infrared Spectrosc., Ed by S.B. Engelsen, K.M. Sørensen and F. van den

Berg. IM Publications Open, Chichester, pp. 11-13 (2019). https://doi.

org/10.1255/nir2017.011

\section{(C) 2019 The Authors}

This licence permits you to use, share, copy and redistribute the paper in any medium or any format provided that a full citation to the original paper is given.

ISBN: 978-1-906715-27-4 
Table 1. Reference data of calibration and test set.

\begin{tabular}{|l|c|c|c|c|}
\hline & \multicolumn{2}{|c|}{ Calibration } & \multicolumn{2}{c|}{ Test set } \\
\hline Parameter & Moisture \% & Crude protein \%dm & Moisture \% & Crude protein \%dm \\
\hline N & 224 & 224 & 46 & 46 \\
\hline Min & 9.6 & 9.7 & 10.7 & 10.1 \\
\hline Max & 14.5 & 17.1 & 13.4 & 14.4 \\
\hline AVG & 11.5 & 13.5 & 12.2 & 12.7 \\
\hline SD & 0.9 & 1.3 & 0.7 & 1.2 \\
\hline
\end{tabular}

geometry, internal reference (black and white) and scanning area: about $2 \mathrm{~cm}^{2}$ for the Extreme and about $1 \mathrm{~cm}^{2}$ for the Auroranir. The Corona Extreme is controlled by an external PC and has been designed for process analysis in agriculture. Auroranir is a handheld device designed for on farm analysis, controlled by an embedded tablet PC and battery operated. Wheat samples were scanned side by side with the two diode array instruments and with the scanning monochromator for reference values.

\section{Software and chemometric treatments}

All spectra were processed using Ucal software ver. 3.0 (Unity Scientific LLC, USA). Two different calibration transfer algorithms were tested: zero order correction and piecewise direct standardization. The mathematical pretreatments of the spectra were standard normal variate (SNV) and detrending for scatter correction and first derivative with a gap and smoothing over four data points for baseline correction. Partial least square calibrations for both diode array instruments were optimized with cross validation and number of principal components.

Calibration transfer performance was evaluated on 46 samples, removed from the original dataset and used for external validation.

\section{Results and discussion}

One of the most used strategies to calibrate portable spectrometers is to transfer the dataset directly from a benchtop spectrometer through various standardization techniques. ${ }^{3}$ In the present trial, it was not possible to transfer directly a laboratory calibration because the scanning monochromator works in a different spectral range, so the Infratec 1241 was used only to provide reference values. Spectra of wheat samples were acquired with the process and the handheld instrument placed side by side, but using different scanning set up. PLS calibrations for both instruments were optimized using crossvalidation and the number of principal components were limited to deal with possible instrument differences. The SECV in calibration was $0.09 \%$ and $0.13 \%$ for moisture and $0.28 \%$ and $0.45 \%$, for protein, respectively for the Auroranir and Corona Extreme. Performance of validation (SEP) within instrument was of $0.07 \%$ and $0.11 \%$ for moisture and of $0.27 \%$ and $0.37 \%$ for protein, for the handheld and the process instrument respectively. The level of accuracy obtained for these parameters is comparable to that obtained by other authors for protein in grain. ${ }^{4-6}$ When the same calibrations were used to predict samples across instruments, without spectral standardization, the SEP was of $0.08 \%$ and $0.19 \%$ for moisture and of $0.34 \%$ and $0.47 \%$ for protein, for Corona Extreme predicting AuroraNIR and vice versa, respectively (Table 2). Ten wheat samples were selected from the calibration dataset and used for computing a zero order correction and a Piecewise Direct Standardization. Only the first method, a simple offset correction, slightly improved the predictions between the two instruments which differed mainly in absorbance due to their different optical geometry.

\section{Conclusions}

The handheld spectrometer slightly outperformed the on line instrument in terms of SECV and SEP within instrument type, probably due to better sampling during the spectral acquisition. Both instruments can accurately predict moisture and protein on wheat and, probably due to a good factory alignment, could use the same calibration avoiding time-consuming calibration transfer procedures. 
Table 2. Calibration and validation performance within and between instruments.

\begin{tabular}{|l|c|c|c|c|}
\hline \multicolumn{1}{|c|}{ Instrument } & \multicolumn{2}{c|}{ Handheld } & \multicolumn{2}{c|}{ Process } \\
\hline Parameter & Moisture \% & Crude protein \%dm & Moisture \% & Crude protein \%dm \\
\hline$P C$ & 5 & 7 & 7 & 6 \\
\hline$R_{\mathrm{CV}}^{2}$ & 0.99 & 0.94 & 0.98 & 0.85 \\
\hline SECV & 0.09 & 0.28 & 0.13 & 0.45 \\
\hline$R_{\text {TSTWI }}^{2}$ & 0.98 & 0.96 & 0.88 & 0.87 \\
\hline SEP & 0.07 & 0.27 & 0.11 & 0.37 \\
\hline$R_{\text {TSTBI }}^{2}$ & 0.89 & 0.82 & 0.98 & 0.95 \\
\hline SEP $_{\mathrm{Bl}}$ & 0.19 & 0.47 & 0.08 & 0.34 \\
\hline
\end{tabular}

$R_{C V}^{2}: R^{2}$ of cross validation; SECV: standard error of cross validation; $\mathrm{SEP}$ w: standard error of prediction within instruments; $\mathrm{SEP}_{\mathrm{B}}$ : standard error of prediction between instruments; $R_{\mathrm{TSTW}}^{2}: R^{2}$ Test set within instruments; $R_{\mathrm{TSTB}}^{2}: R^{2}$ test set between instruments

\section{References}

1. C.A. Teixeira dos Santos, M. Lopo, R.N.M.J. Páscoa and J.A. Lopes, "Review on the applications of portable near-infrared spectrometers in the agro-food industry", Appl. Spectrosc. 67(11), 1215-1233 (2013). https://doi.org/10.1366/13-07228

2. T. Fearn, "Standardisation and calibration transfer for near infrared instruments: a review", J. Near Infrared Spectrosc. 9, 229-244 (2001). https://doi. org/10.1255/jnirs.309

3. A.L. Bizerra Brito, A.V. Pereira Santos, K.D.T. Melo Milanez, M.J. Coelho Pontesa and L.F.B. Lira Pontes, "Calibration transfer of flour NIR spectra between benchtop and portable instruments", Anal. Methods

9, 3184 (2017). https://doi.org/10.1039/c7ay00391a

4. F. Morari et al., "Understanding the effects of sitespecific fertilization on yield and protein content in durum wheat", in Precision Agriculture '13, Ed by J.V. Stafford. Wageningen Academic Publishers, Wageningen (2013).

5. K. Maertens, P. Reyns and J. De Baerdemaeker, "On-line measurement of grain quality with NIR technology", Trans. ASAE 47(4), 1135-1140 (2004). https://doi.org/10.13031/2013.16545

6. D.S. Long, R.E. Engel and M.C. Siemens, "Measuring grain protein concentration with in-line near infrared reflectance spectroscopy", Agron. J. 100, 247-252 (2008). https://doi.org/10.2134/agronj2007.0052 